\title{
A first step towards the understanding of the 5-HT 3 receptor subunit heterogeneity from a computational point of view $\dagger$
}

\author{
Francesca De Rienzo, ${ }^{a b}$ Marta Del Cadia ${ }^{a}$ and Maria Cristina Menziani* ${ }^{a}$ \\ Received 30th March 2012, Accepted 24th July 2012 \\ DOI: $10.1039 / \mathrm{c} 2 \mathrm{cp} 41028 \mathrm{a}$
}

The functional serotonin type-3 receptor $\left(5-\mathrm{HT}_{3}-\mathrm{R}\right)$, which is the target of many neuroactive drugs, is known to be a homopentamer made of five identical subunits $\mathrm{A}\left(5-\mathrm{HT}_{3 \mathrm{~A}} \mathrm{-R}\right)$ or a binary heteropentamer made of subunits $\mathrm{A}$ and $\mathrm{B}\left(5-\mathrm{HT}_{3 \mathrm{~A} / \mathrm{B}}-\mathrm{R}\right)$ with a still debated arrangement and stoichiometry. This complex picture has been recently further complicated by the discovery of additional $5-\mathrm{HT}_{3}-\mathrm{R}$ subunits, $\mathrm{C}, \mathrm{D}$, and $\mathrm{E}$, which, similarly to the B subunit, are apparently able to form functional receptors only if co-expressed with subunit A. Being the binding site for both serotonin and antagonists (i.e. drugs) located at the extracellular interface between two adjacent subunits, the large variability of the $5-\mathrm{HT}_{3}-\mathrm{R}$ composition becomes a crucial issue, since it can originate many different interfaces providing nonequivalent ligand binding sites and complicating the pharmacological modulation. Here, the different $5-\mathrm{HT}_{3}-\mathrm{R}$ interfaces are analysed, on the bases of the structural conformations of previously built $3 \mathrm{D}$ homology models and of the known subunit sequences, by addressing their physicochemical characterization. The results confirm the presence of an aromatic cluster located in the core of the A-A interface as a key determinant for having an interface both stable and functional. This is used as a discriminant to make hypotheses about the capability of all the other possible interfaces constituted by the known $5-\mathrm{HT}_{3}-\mathrm{R}$ sequences $\mathrm{A}, \mathrm{B}, \mathrm{C}, \mathrm{D}$, and $\mathrm{E}$ to build active receptors.

\section{Introduction}

The serotonin type-3 receptor $\left(5-\mathrm{HT}_{3}-\mathrm{R}\right)$ is a cation selective transmembrane protein channel that, together with the nicotinicacetylcholine (nACh), $\gamma$-aminobutyric acid (GABAA) and glycine receptors, belongs to the Cys-loop Ligand-Gated Ion Channel (LGIC) superfamily. 5- $\mathrm{HT}_{3}-\mathrm{R}$ is responsible for fast synaptic transmission at chemical synapses and is the target of many neuroactive drugs. ${ }^{1,2}$ The $3 \mathrm{D}$ structure of $5-\mathrm{HT}_{3}-\mathrm{R}$ has not yet been solved experimentally, however the available data describe a protein system made of five monomers assembled in a pseudosymmetric pentameric shape to form an ion channel permeable to small ions $\left(\mathrm{Na}^{+}, \mathrm{K}^{+}\right) \cdot{ }^{3-5}$ Each monomer contains three domains: an intracellular portion, a transmembrane domain and an extracellular region, where the binding site for serotonin is known to be located. ${ }^{3,6-13}$ The ligand binding site lies at the interface of two adjacent subunits and is formed by the convergence of six so-called "loops": 3,13,14 Loops A, B and C from one of the two subunits, called the principal subunit, and

${ }^{a}$ Department of Chemistry, University of Modena and Reggio Emilia, Via G. Campi 183, 41100 Modena, Italy.

E-mail: mariacristina.menziani@unimore.it; Fax: + 39 059373543; Tel: +390592055091

${ }^{b}$ Center S3 Institute Nanoscience-CNR, Via Campi $213 \mathrm{~A}$,

41125 Modena, Italy.E-mail: francesca.derienzo@unimore.it

$\dagger$ Electronic supplementary information (ESI) available: details of any supplementary information available should be included here. See DOI: $10.1039 / \mathrm{c} 2 \mathrm{cp} 41028 \mathrm{a}$
"Loops" D, E and F in the second subunit, called the complementary subunit. Interestingly, one of these loop moieties, Loop $\mathrm{C}$, which surrounds the binding cavity, undergoes large conformational changes and, therefore, it has been suggested to behave like a lid, opening and closing the access to the binding site. ${ }^{8}$ The conformation assumed by Loop $\mathrm{C}$ seems to be correlated to the overall conformational state of the receptor: the nACh-R assumes a lid-closed local conformation in its active form, ${ }^{8}$ while it seems to prefer a lid-open conformation when bound to inhibitors.

Five different 5- $\mathrm{HT}_{3}-\mathrm{R}$ subunits have been identified so far: subunits $\mathrm{A}$ and $\mathrm{B}$, which have been largely studied and characterized, and subunits $\mathrm{C}, \mathrm{D}$, and $\mathrm{E}^{3,15}$ The subunit $5-\mathrm{HT}_{3}-\mathrm{R} \mathrm{A}$ is capable of forming functional homopentameric receptors; in contrast, $5-\mathrm{HT}_{3}-\mathrm{R} \mathrm{B}$ is unable to express as a homopentamer. However, if coexpressed with subunit 5- $\mathrm{HT}_{3}-\mathrm{R} \mathrm{A}$ it was found to build functional $\mathrm{B} / \mathrm{A}$ heteropentamers. Recent experimental findings point out the great relevance of the $5-\mathrm{HT}_{3} \mathrm{~B} / \mathrm{A}$ receptor in mammalian central and peripheral nervous systems. ${ }^{16}$ The subunit stoichiometry in the heteropentameric receptor is still a matter of debate: recent AFM studies have demonstrated that the heteropentamer has a 2A : $3 \mathrm{~B}$ stoichiometry with a BBABA arrangement, ${ }^{16}$ where no AA interface is present; while the work by Lummis and co-workers, ${ }^{17,18}$ based both on modelling and experimental site directed mutagenesis studies on the $5-\mathrm{HT}_{3}-\mathrm{R}$, supports the 
requirement of at least one AA interface for any $5-\mathrm{HT}_{3}-\mathrm{R}$ to be functional. In particular, they mutated binding-site residues in the mouse $5-\mathrm{HT}_{3}-\mathrm{R}$ A subunit to the corresponding mouse $5-\mathrm{HT}_{3}-\mathrm{R}$ B subunit residues, ${ }^{17}$ and demonstrated that the mutant receptors reduced significantly antagonist-binding affinity and increased 5-HT $\mathrm{EC}_{50}$; in contrast, no significant changes were observed when A-like mutations were introduced into the mouse $5-\mathrm{HT}_{3}-\mathrm{R} \mathrm{B}$ subunit. Therefore, it was suggested that the $5-\mathrm{HT}_{3}-\mathrm{R} \mathrm{B}$ subunit does not contribute to the binding site and $5-\mathrm{HT}_{3} \mathrm{AB}$ receptors presumably contain at least one AA interface.

Thus, the composition of the receptors, by considering different stoichiometries of subunits A and B only, may vary largely, with the possible permutations being: AAAAA; BAAAA; BBAAA; BABAA; BBBAA; BABBA; BBBBA (BBBBB is not considered since it is known not to be expressed). ${ }^{19}$ Although little is known about the functional features of the remaining subunits $\mathrm{C}, \mathrm{D}$, and $\mathrm{E}$ : they are apparently able to form functional heteropentameric receptors if co-expressed with subunit A, as it is also the case of the B subunit. ${ }^{20,21}$ Therefore, the already highly complex picture of $5-\mathrm{HT}_{3}$ receptors stoichiometry is further complicated by the presence of the subunits $\mathrm{C}, \mathrm{D}$, and $\mathrm{E}$ and even more by considering all the subunit sequence isoforms and splice variants. ${ }^{19}$

The many possible different interfaces provide non-equivalent ligand binding sites both for serotonin and for agonists and antagonists, ${ }^{22}$ thus largely affecting the pharmacological modulation of this receptor.

The variability in subunit composition influences agonist response in all the LGICs, such as nAChR, which is the best characterized ion-channel: a 3D low resolution (4 A) structure of this receptor is available (pdb: 2BG9), ${ }^{11}$ and many of its subunit variants have been identified. ${ }^{23,24}$ Other LGIC receptors whose structure has been described are the ELIC (pdb code: 2VL0, $R=3.3 \AA$ ) and GLIC (pdb code: 3EAM, $R=2.9 \AA$ ), which are known in their homomeric form. Ortells and Barrantes ${ }^{25}$ have recently tackled the problem of the assembly mechanism of the LGICs by analysing the complementarity in shape and physical-chemical properties of the various subunit interfaces. Although they briefly take into account $5-\mathrm{HT}_{3}-\mathrm{R} A$ and $\mathrm{B}$, their work is mainly focussed on the detailed analysis of various $\mathrm{nACh}-\mathrm{R}$ subunits in the attempt to predict whether the $\beta 1 \delta \alpha 1 \gamma \alpha 1$ receptor assembles following the so-called "heterodimer model" (which proposes that two dimers $(\delta \alpha 1$ and $\gamma \alpha 1$ ) are formed independently and then associate with each other and with the remaining $\beta 1$ subunit) or the alternative "sequential model" (which hypothesizes that first heterodimers of different compositions are assembled to rapidly form the $\alpha 1 \beta 1 \gamma$ trimer and subsequently add the $\delta$ and the second $\alpha 1$ subunit).

Recently, the $5-\mathrm{HT}_{3}-\mathrm{R} \mathrm{A}-\mathrm{A}$ interface has been analysed and characterised using a Computer Alanine Scanning Mutagenesis (CASM) protocol flanked by MD simulations. ${ }^{26}$ The combined MD-CASM protocol proved to be useful to point out the structurally and energetically critical points at the receptor interface, whose perturbation by ligands might promote or impair the channel activation or the protein functionality. In particular, the presence of an aromatic cluster was pointed out, which is formed by residues $\mathbf{W 1 7 8}$ (hot spot in the principal subunit), and Y83 (hot spot in the complementary subunit),
Y68, W85 and Y148 (warm spot in the complementary subunit) and is located in the middle of the binding interface. This is the "hot centre" of the protein-protein interaction and is probably involved in the correct assembling of the extracellular part of the receptor. In addition, analysis of the coupling of agonis$\mathrm{t}$-antagonist binding to channel activation/inactivation suggested that two of these hot-centre residues, i.e. W178 and Y148, are key points of the binding/activation mechanism.

In this paper, we further study the $5-\mathrm{HT}_{3}-\mathrm{R}$ interfaces by addressing the physicochemical characterization (in particular, the hydrophobic and electrostatic properties) of the many different possible interfaces that can form the $5-\mathrm{HT}_{3}-\mathrm{Rs}$, with the aim to predict, on atomistic bases, the role played by the extracellular moieties of the A and B subunits in the formation of functional or non-functional receptors. The conclusions and hypotheses advanced are further extended to the additional C, D, and E subunits.

\section{Computational details}

\subsection{The subunit-subunit interfaces}

The $5-\mathrm{HT}_{3 \mathrm{~A}}-\mathrm{R}$ (homomeric) and $5-\mathrm{HT}_{3 \mathrm{~A} / \mathrm{B}}-\mathrm{R}$ (heteromeric) structures built and minimized as described by Barbosa et al. ${ }^{27}$ were used to extract the following subunit interfaces: $\mathrm{A}-\mathrm{A}$ (from the homopentamer), A-B, B-A and B-B (from the heteropentamer).

Briefly, homology models of the sequences of the extracellular portion of the human 5- $\mathrm{HT}_{3}-\mathrm{R}$ subunit A (UniProtKB entry: P46098) and subunit B (UniProtKB entry: O95264) ${ }^{28}$ were built using as a template the available structure of nACh-R from Torpedo marmorata, ${ }^{11}$ which has a sequence identity (SeqId) of $27 \%$ and $25 \%$ for subunit $\mathrm{A}$ and $\mathrm{B}$, respectively. The selected template structure (PDB entry: 2BG9, subunit a) is in the so called "open-lid" conformation, characterized by a "closed" conformation of loop C. Loop C has been suggested to behave as a lid, opening and closing the access to the site. ${ }^{8}$ The nACh-R assumes a lid-closed local conformation in its active form, ${ }^{8}$ while it seems to prefer a lid-open conformation when bound to inhibitors: the open-lid conformation of the ligand-free binding interfaces in the nACh-R structure represents the closed state of the ionophore stabilized by antagonists. $^{29}$ Pairwise sequence alignment of the $5-\mathrm{HT}_{3}-\mathrm{R}$ $\mathrm{A}$ and the $5-\mathrm{HT}_{3}-\mathrm{R} \mathrm{B}$ sequence against the $\mathrm{nACh}-\mathrm{R}$ sequence was performed by means of Clustal $W^{30,31}$ and afterwards modified accordingly to experimental information. ${ }^{6}$ Modelling was performed with the software MODELLER v. 8.2.32 results were evaluated with PROCHECK, ${ }^{33}$ QUANTA, ${ }^{34}$ MODELLER $^{32}$ and WhatIf, ${ }^{35,36}$ and assessed against the available structural experimental information. ${ }^{13,14}$ The known pentameric structural arrangement of the nACh-R was used for assembling both the 5- $\mathrm{HT}_{3} \mathrm{~A}-\mathrm{R}$ homopentamer and the 5- $\mathrm{HT}_{3} \mathrm{~A} / \mathrm{B}-\mathrm{R}$ heteropentamer (according to the BBABA pattern subunit assembling), which were then optimised (50 steps of steepest descent followed by conjugate gradient until the convergence to $0.05 \mathrm{kcal} \mathrm{mol}^{-1}$ ) with CHARMm, ${ }^{37}$ using the parameters in the CHARMm22 force field ${ }^{38}$ and a solvent dielectric constant of 5 .

Here and in the following paragraphs, the uppercase letters $\mathrm{A}, \mathrm{B}, \mathrm{C}, \mathrm{D}$ and $\mathrm{E}$ are used to identify the different $5-\mathrm{HT}_{3}-\mathrm{R}$ 
subunit types, while bold lowercase letters $\mathbf{a}, \mathbf{b}, \mathbf{c}, \mathbf{d}$ and $\mathbf{e}$ are used to name and differentiate the five monomers assembled in both the homopentamer and the heteropentamer. The homopentamer $5-\mathrm{HT}_{3 \mathrm{~A}}-\mathrm{R}$ is composed of five identical subunits $\mathrm{A}$, which are named a, b, c, d, and e. Similarly, the heteropentamer $5-\mathrm{HT}_{3 \mathrm{~A} / \mathrm{B}}-\mathrm{R}$ is composed of both A and B subunits (with the stoichiometry BABBA described by Barrera et al. ${ }^{16}$ ), which are named $\mathbf{a}, \mathbf{b}, \mathbf{c}, \mathbf{d}$, and e. A homodimer $\mathrm{A}-\mathrm{A}$ composed of the adjacent subunits identified as monomers a and $\mathbf{e}$ was then extracted from the minimized homopentamer and used in further analysis. Similarly, the heterodimers $\mathbf{a}-\mathbf{b}$ and $\mathbf{d}-\mathbf{c}, \mathbf{c}-\mathbf{a}$ and $\mathbf{e}-\mathbf{d}$, and the homodimer b-e were extracted from the heteropentamer to analyze the A-B, $\mathrm{B}-\mathrm{A}$ and $\mathrm{B}-\mathrm{B}$ interface, respectively, and to compare them with the $\mathrm{A}-\mathrm{A}$ interface.

\subsection{Calculation of physical-chemical parameters relevant for protein-protein interaction}

The physico-chemical study of the dimer interface surfaces was performed using the MolSurfer package, ${ }^{39-41}$ which includes the analysis of the electrostatic, hydrophobic and shape complementarity.

Using as input the individual structure of each monomer in the dimer, the MolSurfer package generates 3D surface-surface interface maps which are then projected on a $2 \mathrm{D}$ space. The interface between the two macromolecules (protein-protein) is mapped by Adsi, ${ }^{39-41}$ and the molecular properties of each protein are projected onto every point of the interface (these are the properties assigned to the atom closest to the point). The interface between the macromolecules is considered a contact surface if the surface-to-surface distance is less than $5 \AA$ (considering van der Waals surfaces of the two subunits). The values of the surface-to-surface distances, hydrophobicities and electrostatic potentials are displayed at each point of the interface surface. Surface to surface distance is an indicator of the interface shape complementarity.

The molecular electrostatic potential (MEP) of each isolated protein was computed with the $\mathrm{UHBD}^{42}$ program by solving the finite difference linearized Poisson-Boltzmann equation and the potential values were interpolated at each interface point. The parameters used are: the Amber force field to describe atomic radii and charges, ${ }^{43,44}$ a grid dimension of $99 \times 99 \times 99 \AA^{3}$ with $1 \AA$ grid spacing, an ionic strength of $150 \mathrm{mM}$, a protein dielectric constant of 5 and a solvent dielectric constant of 80 , an ionic radius of $1.5 \AA$ and the van der Waals surface to separate the protein and the solvent. Residue hydrophobicities were assigned according to the residue name and the parameters in Eisenberg et al. ${ }^{45}$

Besides the hydrophobicities and the electrostatic potentials, also their similarity indices, namely HSI (Hydrophobicity Similarity Index) and EPSI (Electrostatic Potential Similarity Index), are computed by MolSurfer and mapped onto the 2D projection of the dimer interface. ${ }^{39-41}$

Electrostatic complementarity at the 5- $\mathrm{HT}_{3}-\mathrm{R}$ dimer interfaces is quantified by negative values (EPSI between 0 and -1 ; red areas) of the electrostatic potentials similarity index: the more negative is the EPSI, the higher is the surface electrostatic potential (EP) complementarity. In fact, a negative EPSI indicates that the residues on the two interacting protein surfaces are characterized by opposite charged areas, i.e. there are positive residues on one surface and negative residues on the other surface. A positive value of EPSI (blue areas on the 2D EPSI map) indicates instead that the residues on the two surfaces are similarly charged (which means no electrostatic complementarity). In contrast, the hydrophobic complementarity at the dimer interface is higher when the HSI assumes positive values (blue areas on the 2D map): this in fact means that the hydrophobic properties of the two interacting protein surfaces are similar, which is a determinant requirement for hydrophobic complementarity. Negative values (corresponding to red areas on the 2D maps) instead indicate that the two surfaces have different hydrophobic properties (no hydrophobic complementary).

\subsection{Sequence alignments}

The sequences for the $5-\mathrm{HT}_{3}-\mathrm{R}$ subunit $\mathrm{C}, \mathrm{D}$, and $\mathrm{E}$ were extracted from the UniProt/SwissProt databank with UniProtKB accession codes: Q8WXA8 (subunit C), Q70Z44 (subunit D), and A5X5Y0 (subunit E). The multiple alignment of the five 5- $\mathrm{HT}_{3}-\mathrm{R}$ subunits was obtained with Clustal $\mathrm{W}$ at the EBI (internet address: http://www.ebi.ac.uk/Tools/msa/clustalw2/) using the BLOSUM matrix, a gap pen penalty of 10 , a gap extension penalty of 0.20 , and a gap distance penalty of 5 . This alignment is shown in Fig. 1 together with sequence similarity percentages.

\section{Results}

\subsection{Analysis of the $5-\mathrm{HT}_{3}-\mathrm{R}$ homo- and heteropentameric folds}

As described in the Computational details section, both the $5-\mathrm{HT}_{3}-\mathrm{R} \mathrm{A}$ and $\mathrm{B}$ subunits were modelled using the subunit $\mathbf{a}$ in the $\mathrm{nAChR}$ structure (pdb: 2BG9) as a template. In this structure, Loop $\mathrm{C}$ is in the open conformation (see Fig. 2), therefore in the 5-HT $-\mathrm{H}_{3} \mathrm{R}$ A and B models Loop C is also opened and the homoand heteropentamers initially assembled are formed by subunits with Loop $\mathrm{C}$ in the open conformation. Interestingly, after the minimization procedure, the starting conformation changes (Fig. 2). In the homopentameric 5- $\mathrm{HT}_{3 \mathrm{~A}}-\mathrm{R}$, all the subunits show a Loop $\mathrm{C}$ in its closed conformation; in contrast, in the BBABA heteropentamer, different subunits assume different conformations. In fact, subunits $\mathrm{A}$ have their Loop $\mathrm{C}$ in its closed conformation (independently of their complementary subunit), while all subunits B have their Loop C in its open conformation (independently of their complementary subunit): therefore, dimers A-B have their principal subunit Loop $\mathrm{C}$ closed as the homopentameric A-A dimers, while dimers B-A have their principal subunit Loop $\mathrm{C}$ open as the dimers B-B. This is schematized in Fig. 2.

Since Loop $\mathrm{C}$ is closed when the channel is open (i.e. functional) and, vice versa, Loop $\mathrm{C}$ is open when the channel is closed (non-functional), these findings lead to the preliminary hypothesis that the $\mathrm{A}-\mathrm{A}$ and the $\mathrm{A}-\mathrm{B}$ interfaces are functional while the $\mathrm{B}-\mathrm{B}$ and $\mathrm{B}-\mathrm{A}$ interfaces are not.

\subsection{Long range: surface molecular electrostatics of the $A$ and $B$ subunits and $\mathrm{AA}, \mathrm{BB}, \mathrm{AB}$ and $\mathrm{BA}$ dimers}

Electrostatics are determinants since they are the driving forces for long range interactions and complex assembling. At short range, the effect of the many dispersion interactions 
a) $h 5 H T 3 R \quad A \mid 24-241$ h5HT3R B 22-238 h5HT3R C| 28-248 h5HT3R D 25-232 h5HT3R E| 26-248

h5HT3A SSPr: h5HT3B SSPr: h5HT3C SSPr: h5HT3D SSPr. h5HT3E SSPr:

h5HT3R A | 24-241 h5HT3R B 22-238 h5HT3R C| 28-248 h5HT3R D 25-232 h5HT3R E|26-248

h5HT3A ssPr: h5HT3B ssPr: h5HT3C SsPr: h5HT3D SSPr: h5HT3E SSPr:

h5HT3R A| 24-241 \begin{tabular}{ll|l} 
h5HT3R & B & $22-238$
\end{tabular} h5HT3R C|28-248 h5HT3R D|25-232 h5HT3R E|26-248

h5HT3A ssPr: h5HT3B ssPr: h5HT3C SSPr: h5HT3D SSPr: h5HT3E SSPr:

h5HT3R A| 24-241 h5HT3R B 22-238 h5HT3R C| 28-248 h5HT3R D 25-232 h5HT3R E| 26-248

h5HT3A SSPr: h5HT3B ssPr: h5HT3C ssPr: h5HT3D ssPr: h5HT3E ssPr:
- - RRSRNTTRPALLRLSDYLLTNYRKGVRPVRDWRKPTTVSIDVIVYAILNVDEKNQVL 80 - - TDTHHPQDSALYHLSKQLLQKYHKEVRPVYNWTKATTVYLDLFVHAILDVDAENQIL 78 - -FTINCSGFDQHGVDPAVFQAVFDRKAFRPFTNYSIPTRVNISFTLSAILGVDAQLQLL 85 DTLIINCPGFGQHRVDPAAFQAVFDRKAIGPVTNYSVATHVNISFTLSAIWNCYSRIHTF 84 VTFTINCSGFGQHGADPTALNSVFNRKPFRPVTNISVPTQVNISFAMSAILDVNEQLHLL 85

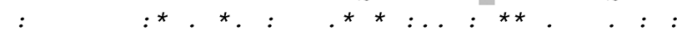
- - CCCCCCCCHHHHHHHHHHHHCCCCCCCCCCCCCCCEEEEEEEEEEEEEECCCCCEEE - - CCCCCCCCHHHHHHHHHHHHCCCCCCCCCCCCCCCEEEEEEEEEEEEECCCCCCCEE - - CCCCCCCCCCCCCHHHHHHHHCCCCCCCCCCCCCCCEEEEEEEEEEEEECCCCCCCEE CCEE E ECCCCCCCCHHHHHHHCCCCCCCCCCCCCCEEEEEEE EEEEEEE ECCCCCCEE CEEEEECCCCCCCCCHHHHHHHHCCCCCCCCCCCCCCCEEEEEEEEEEEEECCCCCCEEE

TTYIWYRQYWTDEFLQWNPEDFDNITKLSIPTDSIWVPDILINEFVDVGKSPNIPYVYIR 140 KTSVWYQEVWNDEFLSWNSSMFDEIREISLPLSAIWAPDIIINEFVDIERYPDLPYVYVN 138 TSFLWMDLVWDNPF INWNPKECVGINKLTVLAENLWLPDIFIVESMDVDQTPSGLTAYIS 145 N--CHHARPWHNQFVQWNPDECGGIKKSGMATENLWLSDVFIEES - -VDQTPAGLMASMS 140 SSFLWLEMVWDNPFISWNPEECEGITKMSMAAKNLWLPDIFIIELMDVDKTPKGLTAYVS 145

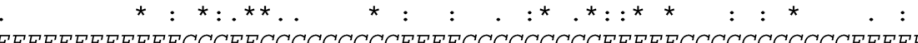
政 CCEEEEECEEECCCCEECCCCCCCCCEEEECCCCCCCCCEEEEECCCCCCCCCCCEEEEE EEEEEEEEEE ECCCEECCCCCCCCCEEEECCCCCCCCCEEEEECCCCCCCCCCCEEEEE E- - EEEEEEEECCCEEECCCCCCCEEEEEECCCCCCCCCEEEEEC- CCCCCCCCEEEEE EEEEEEEEEEE ECCEECCCCCCCCCEEEECCCCCCCCCEEEEECCCCCCCCCCCEEEE

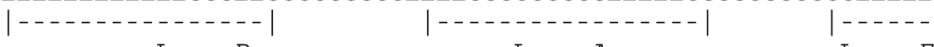
Loop $D \quad$ Loop $A$ Loop $E$

HQGEVQNYKPLQVVTACSLDIYNFPFDVQNCSLTFTSWLHTIQDINISLWRLPEKVKSDR 200 SSGTIENYKPIQVVSACSLETYAFPFDVQNCSLTFKSILHTVEDVDLAFLRSPEDIQHDK 198 SEGR I KYDKPMRVTS ICNLDIFYFPFDQONCTFTFSSFLYTVDSMLLGMDKEVWEITDTS 205 - - - - IVKA TSNTISQCGWS - - - - ASANWTPSISPSMD-RGRAWRRMSRSFQIHHRTS 190 NEGRIRYKKPMKVDSICNLDIFYFPFDQQNCTLTFSSFLYTVDSMLLDMEKEVWEITDAS 204

.. *

$\star \quad: \quad: \quad . \quad:$ .

CCEEEEEECCEEEEEECEEEEEECCCCCCEEEEEEEEEEEECCEEEEEECCCCCCCCCCC CCEEEEEECCEEEEECCEEEEEEECCCCEEEEEEEEEEEECCCEEEEEECCCCCCCCCCC CCCEEEEECCEEEEEEEEEEEEEECCCCEEEEEEEECEEECCCEEEEEECCCCCCCCCCC - - - - EEEECCCEEEEEEEE- - - - EEEECCCCCCEEEE-EEEECEECCCEECCCCCCC CCCEEEEECCCEEEEEEEEECCCCCCCCEEEEEEEECEEECCCEEEEEECCCCHHHCCCC $------\mid$

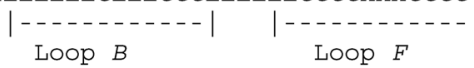

-SVFMNQGEWELLGVLPYFREFSMESSNYYAEMKFYVVIRRR- - - - - 241 -KAFLNDSEWELLSVSSTYSILQS - SAGGFAQIQFNVVMRRH- - - - - 238 RKVIQTQGEWELLGINKATPKMSM-GNNLYDQIMFYVAIRR--RPS- - 248 - - FRTRREWVLLGIQKRTIKVTV-ATNQYEQAIFHVAIRRRCRPSPY 232 RNILQTHGEWELLGLSKATAKLSR-GGNLYDQIVFYVAIRR--RPS- - 248

$$
\text { : . ***. : . . . : : * * . :* }
$$

- CCCEECCCEEEEEECCCEEEEECCCCCCCEEEEEEEEEEEC - . . -

- CCCEECCCEEEEEEEEEEEEECC-CCCCCEEEEEEEEEEEC- - - - CCCCEECCCEEEEEECCCCCCCCC-CCCCCCEEEEEEEEEE--CCC-- - - CCCCCCEEEEEEEEEEEEECC-CCCCCEEEEEEEEEEECCCCCCC CCCCCCCCCEEEEEEECCEEEECC-CCCCCCEEEEEEEEEE--CCC-|-.-.-.-.--n|

$$
\text { Loop C }
$$

\begin{tabular}{cccccc}
\multicolumn{5}{c}{ Sequence similarity } & \multicolumn{4}{c}{ percentage } \\
& A & B & C & D & E \\
A & 100 & & & & \\
B & 48 & 100 & & & \\
C & 35 & 30 & 100 & & \\
D & 21 & 15 & 42 & 100 & \\
E & 35 & 32 & 74 & 39 & 100
\end{tabular}

Fig. 1 ClustalW multiple sequence alignment of the five 5- $\mathrm{HT}_{3}-\mathrm{R}$ subunits $\mathrm{A}, \mathrm{B}, \mathrm{C}, \mathrm{D}$ and $\mathrm{E}$. The prediction of the secondary structure is reported for all the five sequences: $\mathrm{C}=$ random structure; $\mathrm{H}=\alpha$-helix; $\mathrm{E}=\beta$-strand. The sequence similarities are also reported. Gray underlined bold characters indicate the residues forming the "hot-core" of the protein interface. Gray bold italic characters indicate the residue P150, known to be important for protein function. Code: $*=$ conserved residue in all sequences; : = different but highly conserved (very similar) residues; . = different residues that are somewhat similar.

(well represented by hydrophobicity and shape complementarity) becomes dominant to finely tune protein-protein association, while the role of electrostatics is that of providing key point of specific interaction on the surface.

The molecular electrostatic potentials (MEPs) of the 5- $\mathrm{HT}_{3}-\mathrm{R}$ $A$ and $B$ extracellular subunits are shown in Fig. 3. Here, the
MEPs of both the principal and the complementary interface are depicted for subunits $\mathrm{A}$ and $\mathrm{B}$. It is evident that while the principal interface of subunit $\mathrm{A}$ is mainly negative, its complementary side is mainly positive. In contrast, both the principal and the complementary interfaces of subunit B appear to be mainly negative. This MEP distribution has consequences 


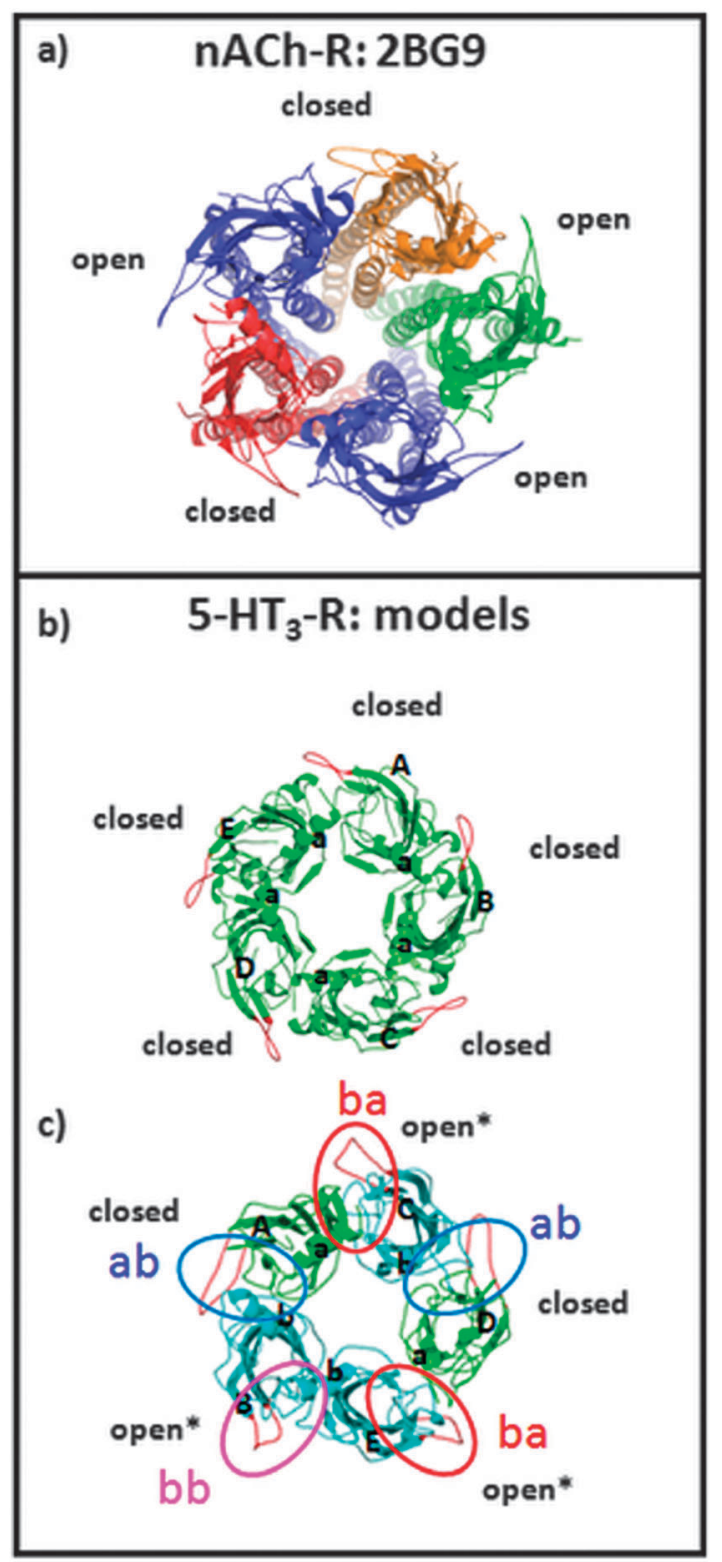

Fig. 2 Cartoon representation of (a) the pentameric structure of nAChR (pdb entry: 2bg9), (b) the minimized homopentameric $5-\mathrm{HT}_{3 \mathrm{~A}}-\mathrm{R}$ model, (c) the minimized heteropentameric $5-\mathrm{HT}_{3 \mathrm{~A} / \mathrm{B}}-\mathrm{R}$ model (stoichiometry BBABA). Code in (a): blue: $\alpha_{1}$ subunit; red: $\gamma$ subunit; green: $\delta$ subunit; yellow: $\varepsilon$ subunit. Code in (b) and (c): light green: subunit 5-HT $-\mathrm{R} A$; cyan: subunit 5- $\mathrm{HT}_{3}-\mathrm{R}$ B; orange: Loop $\mathrm{C}$; blue: $\mathrm{A}-\mathrm{B}$ interface; red: $\mathrm{B}-\mathrm{A}$ interface; pink: $\mathrm{B}-\mathrm{B}$ interface; black uppercase letters: indicate the 5 - $\mathrm{HT}_{3}-\mathrm{R}$ subunit type in the $5-\mathrm{HT}_{3}-\mathrm{R}$ pentamer; black lowercase letters: indicate the name of each monomer. Loop C code: open: Loop C is its open conformation; closed: Loop C is in its closed conformation; open*: the loop is in an open conformation which differ from that before optimization.

on the types of dimers that can be assembled: the electrostatics will preferentially drive proteins with positive patches towards proteins with negative patches and vice versa. For example: the A secondary interface may interact with the A principal interface or with the B principal interface to form the AA dimer or the BA dimer respectively. This does not mean that only the AA and BA dimers can be formed but that those dimers are more probable to assemble than the others.
The analysis of the MEP distribution on the dimer surfaces showed (Fig. 3) that: dimer AA has a complementary interface which is positive and a principal one which is negative; dimer $\mathrm{AB}$ has a complementary interface which is positive and a principal interface which is negative, similarly to the AA dimer; the dimers BA and BB show a similar MEP distribution with both the complementary and the principal interfaces being mainly negative.

Finally, also the MEPs of three 5- $\mathrm{HT}_{3}$ - R pentamers (Fig. 3), i.e. the homopentamer $\mathrm{A}$, the heteropentamer $3 \mathrm{~B}: 2 \mathrm{~A}$ (with a BBABA stoichiometry), the heteropentamer 3A : 2B (with a ABABA stoichiometry), are shown. Interestingly, the surfaces of the two heteropentamers appear highly similar and both their pores are highly negatively charged, as it would be required to transport a positive ion $(\mathrm{Na}+)$. In contrast, the homopentamer A has a central pore which alternates positive and negative sites. This preliminary description is in agreement with previous experimental data showing that the single-channel conductance is higher in the heteropentamer $\mathrm{AB}$ than in the homopentamer A. ${ }^{16}$

\subsection{Short range: physico-chemical and structural analysis of the interfaces $\mathbf{A}-\mathbf{A}, \mathbf{A}-\mathbf{B}, \mathbf{B}-\mathbf{A}$ and $\mathbf{B}-\mathbf{B}$}

All the interfaces analysed are shape-complementary (their atom to atom distance ranges from 2.9 to $6.8 \AA$ ), therefore hereafter this index will not be analysed in detail, while the attention will be focussed on the description and analysis of the electrostatic potentials (EPs) and the hydrophobicities $(\mathrm{H})$ of the dimer interfaces.

3.3.1 The interface $\mathbf{A}-\mathbf{A}$. The $\mathrm{A}-\mathrm{A}$ dimer was extracted from the homopentameric 5-HT $3 \mathrm{~A}-\mathrm{R}$. The electrostatic potential similarity index (EPSI) map shows that there is a high electrostatic complementarity in the core of the interface (see Fig. 4). In addition, in this area, the hydrophobicity similarity index (HSI) map shows that there are residues on both surfaces with similar hydrophobic properties (see Fig. 4).

Three regions with very high complementary physico-chemical features can be highlighted on the 2D surface:

Zone 1 (maximum value of EPSI in the area $(\max$ EPSI) $=$ -0.999 ; HPSI $=-0.365 /+1)$ : this region comprises the following residues: W178, L179 on the principal subunit; Y148, P150, W85 on the complementary subunit. It is a region rich in aromatic residues which interacts through an extended $\pi-\pi$ stacking and originates an aromatic core. This was previously $^{26}$ identified as the "hot core", i.e. the region responsible for the stabilization of the monomer-monomer interface. In addition, P150 has been previously demonstrated to be directly involved in receptor function, ${ }^{27}$ therefore, it appears to be crucial.

Zone $2(\max$ EPSI $=-0.996 ;$ HPSI $=-0.280 /+0.7)$ : this area is constituted by residue E224 on the principal subunit, and the interacting residue $\mathrm{R} 87$ on the complementary subunit. This is a charge-reinforced $\mathrm{H}$-bond interaction, which keeps Loop $\mathrm{C}$ in the principal subunit bound to the complementary subunit. Such a clear electrostatic complementarity is complemented by a good hydrophobic complementarity (see Fig. 4).

Zone $3(\max$ EPSI $=-0.975 ;$ HPSI $=+0.280 /+1)$ : in this area, the following interacting residues are found: T181, Q183, 


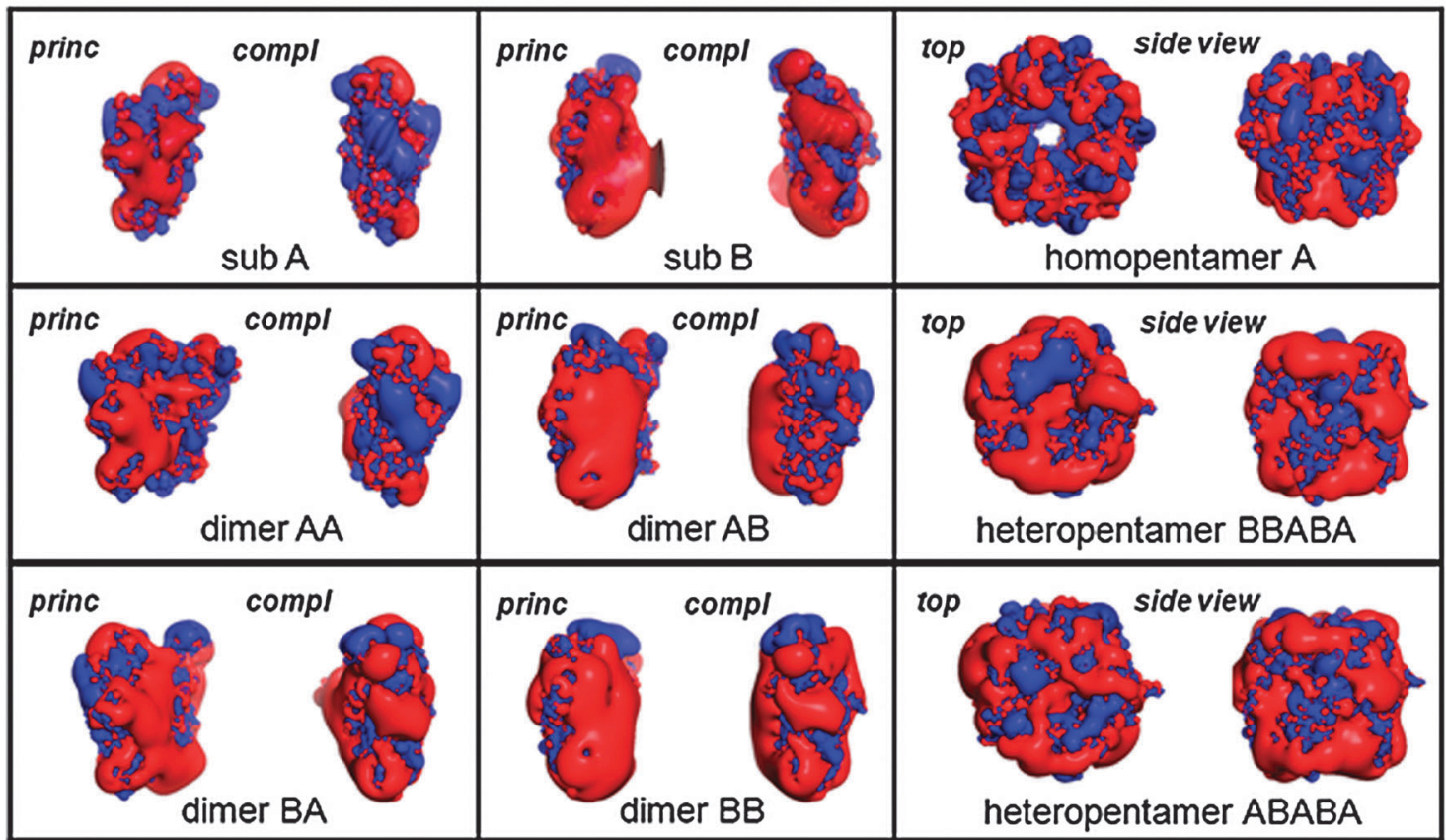

Fig. 3 (Left): representation of MEP isocontour levels at $+1 \mathrm{kT}$ (blue) and $-1 \mathrm{kT}$ (red) of the principal and complementary interface of: top 5- $\mathrm{HT}_{3}-\mathrm{R}$ subunits A and $\mathrm{B}$; middle and bottom $-5-\mathrm{HT}_{3}-\mathrm{R}$ dimers $\mathrm{AA}, \mathrm{BB}, \mathrm{AB}$ and $\mathrm{BA}$. (Right) MEP isocontour levels at $+1 \mathrm{kT}$ (blue) and $-1 \mathrm{kT}$ (red) of the side and top view of: top $-5-\mathrm{HT}_{3}-\mathrm{R}$ homopentamer $\mathrm{A}$; middle $-5-\mathrm{HT}_{3}-\mathrm{R}$ heteropentamer BBABA; bottom $-5-\mathrm{HT}_{3}-\mathrm{R}$ heteropentamer ABABA.

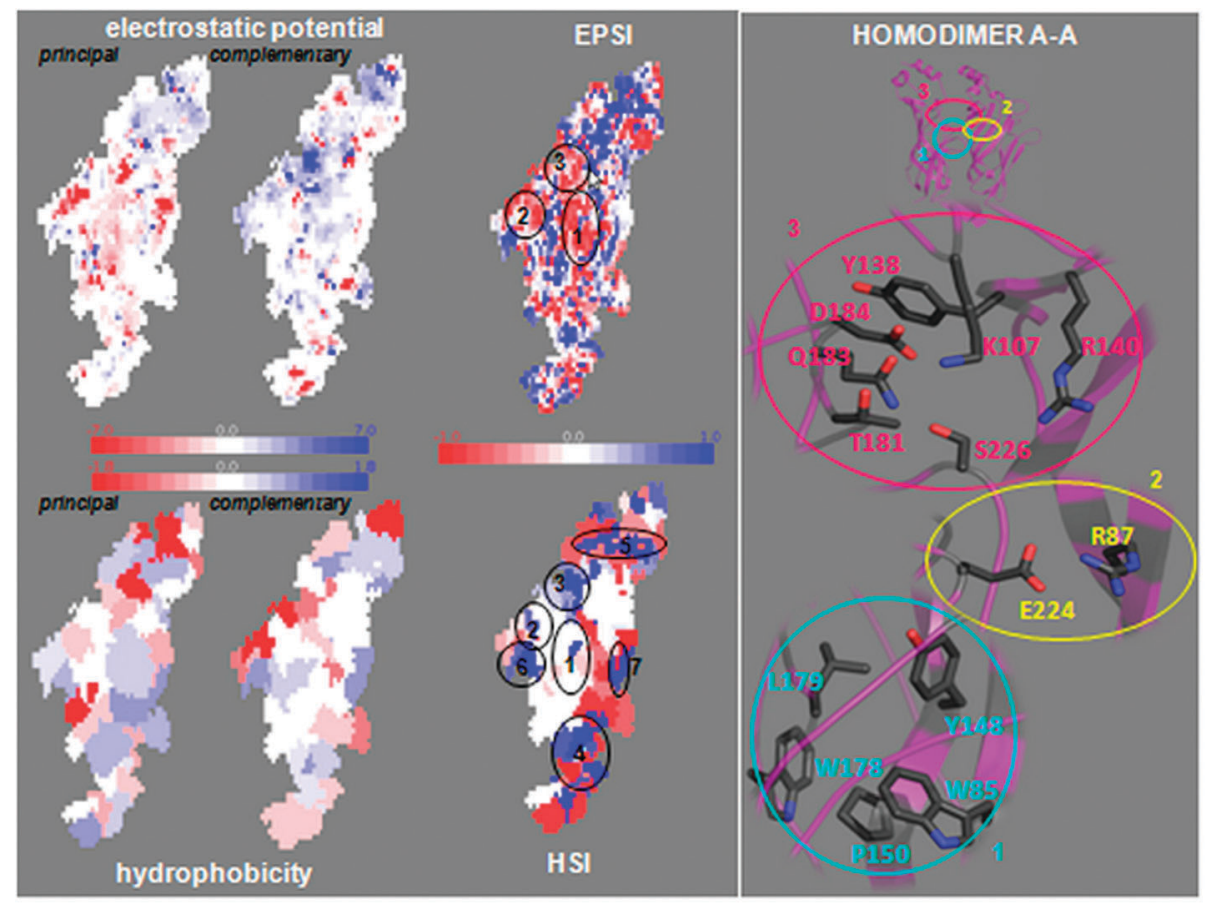

Fig. 4 Homodimeric 5- $\mathrm{HT}_{3}-\mathrm{R}$ A-A interface. (Left) top - 2D maps of the electrostatic potential of the principal subunit and the complementary subunit interfaces and of their similarity (EPSI); bottom - 2D maps of the residue hydrophobicity of the principal subunit and the complementary subunit interfaces and of their similarity (HSI). (Right) top - cartoon representation of the A-A dimer with the interface regions involved in interface stabilization highlighted; bottom - focus on Zones 1-3. Code for the 2D maps: the electrostatic potential ranges from $-7.0 \mathrm{kcal}^{\mathrm{mol}}{ }^{-1} \mathrm{e}^{-1}$ (red; negative potential) to $+7.0 \mathrm{kcal} \mathrm{mol}{ }^{-1} \mathrm{e}^{-1}$ (blue; positive potential); the hydrophobicity ranges from $-1.8 \mathrm{kcal} \mathrm{mol}{ }^{-1}$ (red, hydrophilic) to $+1.8 \mathrm{kcal} \mathrm{mol}^{-1}$ (blue, hydrophobic); EPSI ranges between -1 (red, complementary EPs) and +1 (blue, non-complementary EPs); HSI ranges between -1 (red, non-complementary hydrophobicities) and +1 (blue, complementary hydrophobicities). Maps were generated using the program MolSurfer. ${ }^{39-41}$ Code for the cartoon representations: cyan: Zone 1; yellow: Zone 2; pink: Zone 3; capital letters and numbers: residues at the principal subunit interface; capital italic letters and numbers: residue at the complementary subunit interface. 
D184, S226 on the principal subunit and K107, Y138, R140 on the complementary subunit. The principal surface is rich in negative and polar residues, while the second one is rich in positive and polar residues: both the electrostatic and hydrophobic complementarity criteria are satisfied.

As it can be clearly observed from Fig. 4 bottom, at the AA interface there are additional areas showing complementary hydrophobicity (see for example, area numbers 4, 5, 6 and 7, where the HSI reaches values of about 1). This indicates that, once the two proteins have been brought into association distance, the binding is tuned by the presence of many local hydrophobic and dispersion interactions which are distributed over the whole interface surface.

3.3.2 The interface $\mathbf{B}-\mathbf{B}$. The $\mathrm{B}-\mathrm{B}$ dimer was extracted from the heteropentamer $5-\mathrm{HT}_{3}-\mathrm{R} \mathrm{A} / \mathrm{B}$ and it is composed of the subunits at positions $\mathbf{b}$ and $\mathbf{e}$ in the pentameric arrangement (Fig. 2c). The physico-chemical properties of interface B-B are depicted in Fig. 5.

The electrostatic complementarity of these two monomers is largely reduced with respect to the $\mathrm{A}-\mathrm{A}$ interface and it is not localized in a specific area of the interface. The hydrophobic complementarity instead is more preserved (see Fig. 4 and 5 for comparison). This might suggest that should the two B subunits eventually come into contact (for example if forced by local spatial restraints), their association is then optimized and maintained by short-range interactions. Here, differently from the $\mathrm{A}-\mathrm{A}$ dimer, Loop $\mathrm{C}$ is open: it does not interact with the complementary subunit.

Zone 1 ( $\max$ EPSI $=-0.998 ;$ HPSI $=-0.782 /+0.99)$ : this interface region is formed by residues I122, E124, F125, V126 in the principal subunit and $\mathbf{W 8 5}, \mathrm{P} 150$ and I151 in the complementary one. Due to its residue composition, the hydrophobic complementarity is low.

The "hot core" observed at the A-A interface is completely missing at this interface: $\mathbf{W 1 7 8}$ in the principal subunit is substituted by His in the modelled structure of the B subunit ${ }^{27}$ and by Ile in the $5-\mathrm{HT}_{3}-\mathrm{R}$ subunit multiple sequence alignment (see alignment in Fig. 1); the hot spot Y83 and Y68 in the complementary subunit are replaced by Ser and His, respectively; W85 in the complementary subunit is present although, in the 3D model, it interacts with E124 (on the principal subunit) instead that forming $\pi$-stacking interactions with other aromatic residues of the "hot aromatic core"; finally, Y148 is also present in the complementary surface, however it is not involved in the $\pi$-stacking interactions, which have been shown to be determinant for the interface stabilization in the A-A dimer.

Zone $2(\max$ EPSI $=-0.98 ;$ HPSI $=0 /+1)$ : here, both the hydrophobic and the electrostatic potential are highly complementary, however it is a very restricted area formed by the unique interaction of Y162 in the principal subunit with N205 in the complementary one.

Zone 3 ( $\max$ EPSI $=-0.997 ;$ HPSI $=-0.6 /+1)$ : an additional area with relatively good complementarity can be highlighted in the interface area which is located towards the N-terminus. Here, the interface is stabilized by the following interactions: R50 (principal subunit) - S109 (complementary subunit); V49 (principal subunit) - I108 (complementary subunit); W55 (principal subunit) I105 (complementary subunit); N54 (principal subunit) - E104 (complementary subunit). These residues are spread out on the two protein surfaces, thus forming a large interface area.

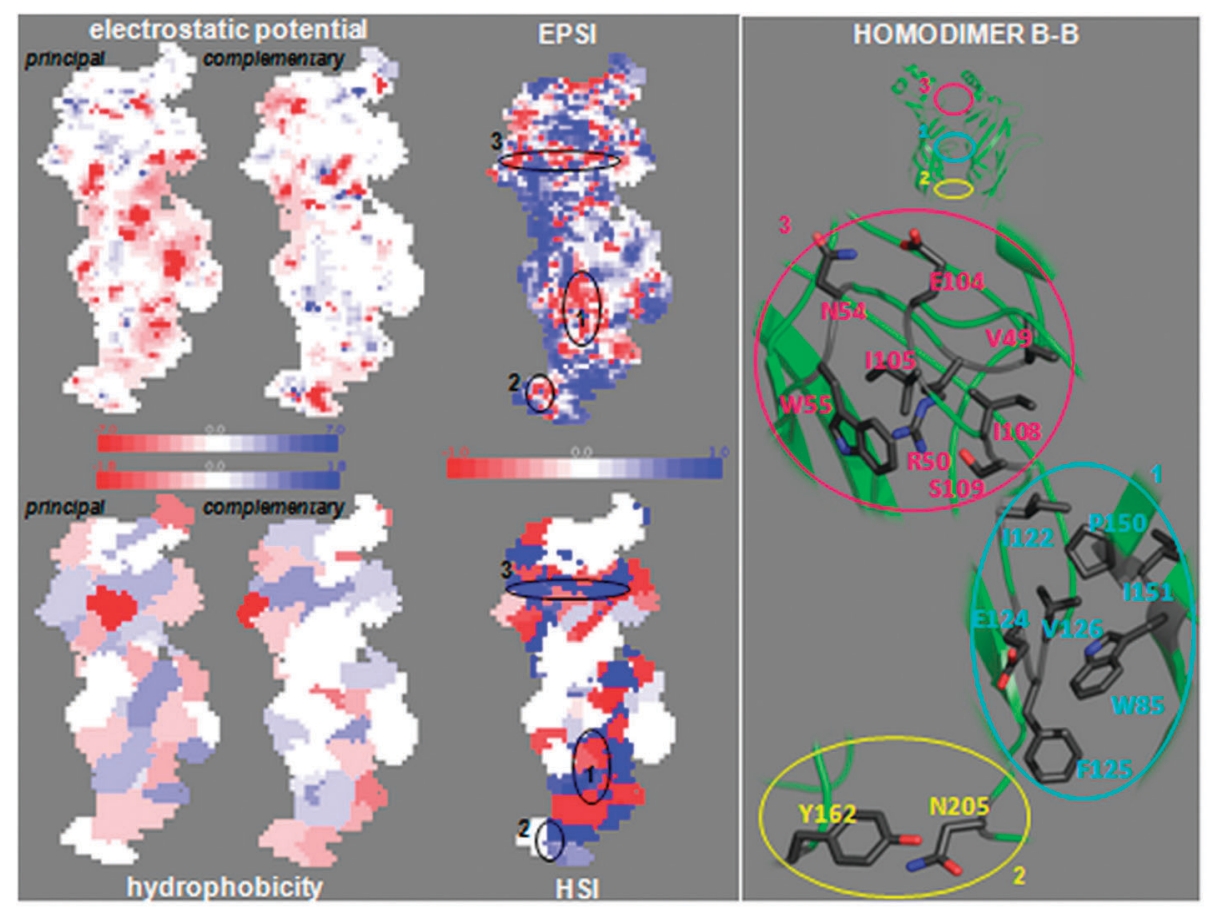

Fig. 5 Homodimeric 5- $\mathrm{HT}_{3}-\mathrm{R}$ B-B interface. (Left) top - 2D maps of the electrostatic potential (EP) of the principal subunit and the complementary subunit interfaces and of their similarity (EPSI); bottom - 2D maps of the residue hydrophobicity of the principal subunit and the complementary subunit interfaces and of their similarity (HSI). (Right) top - cartoon representation of the B-B dimer with the residues determinant for the interface stabilization highlighted; bottom - focus on Zones 1-3. Scheme and colouring codes are the same as those of Fig. 4 . 


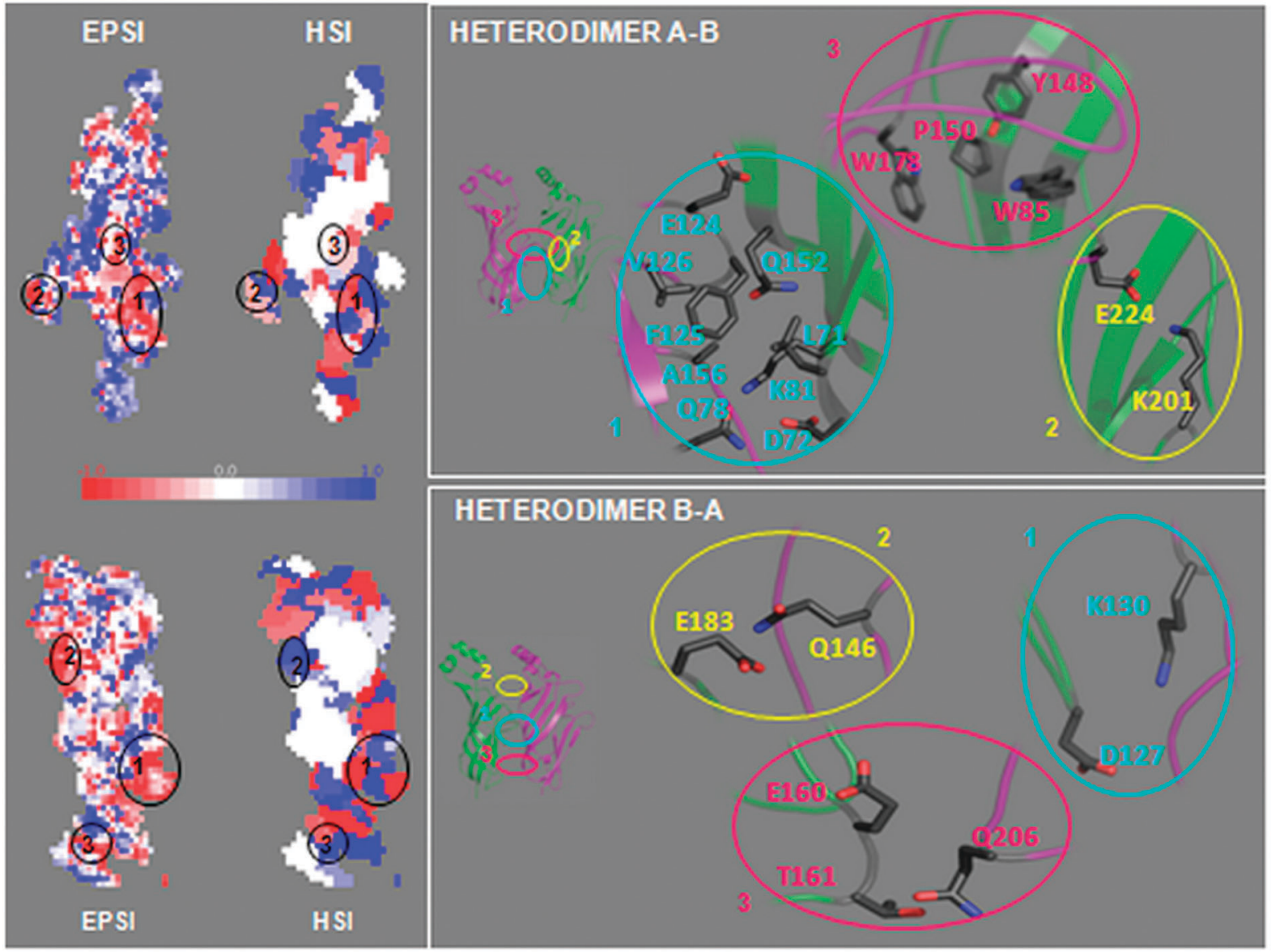

Fig. 6 Heterodimeric 5-HT $-\mathrm{H}$ A-B and B-A interface. (Top) - A-B interface: 2D maps of the electrostatic potential similarity (EPSI) and the hydrophobicity similarity (HSI); cartoon representation of the A-B dimer with the residues determinant for the interface stabilization highlighted (focus on the right). Bottom - B-A interface: 2D maps of the electrostatic potential similarity (EPSI) and the hydrophobicity similarity (HSI); cartoon representation of the B-A dimer with the residues determinant for the interface stabilization highlighted (focus on the right). Scheme and colouring codes are the same as those of Fig. 4.

3.3.3 The interface A-B. The dimer A-B was extracted from the heteromeric $5-\mathrm{HT}_{3 \mathrm{~A} / \mathrm{B}}-\mathrm{R}$ structure. This dimer is characterized by having an $\mathrm{A}$ monomer as the principal subunit and a B monomer as the complementary subunit. Two A-B interfaces exist in the heteropentamer $5-\mathrm{HT}_{3 \mathrm{~A} / \mathrm{B}}-\mathrm{R}$, since an $\mathrm{A}-\mathrm{B}$ interface is formed by the adjacent monomers at positions $\mathbf{a}$ and $\mathbf{b}$ and by the adjacent monomers at positions $\mathbf{d}$ and $\mathbf{c}$ in the pentameric arrangement (Fig. 2b bottom). These two interfaces are highly similar, therefore the analysis was performed using the dimer formed by monomers $\mathbf{a}$ and $\mathbf{b}$. Interestingly, as shown by the maps in Fig. 6, the physicochemical complementarity of the A-B interface is intermediate with respect to that observed for the A-A (Fig. 4) and that observed for the B-B (Fig. 5) interfaces: it is largely reduced with respect to the $\mathrm{A}-\mathrm{A}$ interface and more extended than at the B-B interface. The EP complementarity is displaced towards the dimer interface boundary, both in the region near the membrane and in the opposite region at the channel entrance (see Fig. 6 top), therefore, the residues determining the surface-surface complementarity are different from those in the $\mathrm{A}-\mathrm{A}$ system.

Zone 1 ( $\max$ EPSI $=-0.99$; HPSI $=-0.971 /+1)$ : this area is formed by residues: Q78, A156, E124, F125, V126 in the principal subunits (which is an A monomer) and residues L71, $\mathrm{D} 72, \mathrm{~K} 81, \mathrm{Q} 152$ in the complementary subunit which is a B monomer.

Zone $2(\max$ EPSI $=-0.99 ;$ HPSI $=-0.488 /+0.855)$ : residue E224 in the principal subunit interacts with residue $\mathrm{K} 201$ in the complementary one through a charge-reinforced H-bond interaction which helps to keep the Loop $C$ of the complementary subunit in a closed conformation (when Loop $C$ is at this position the channel is open). There are further additional interactions, due to $\pi-\pi$ stacking which maximize the HSI at the expenses of the EPSI.

Zone $3(\max$ EPSI $=-0.987$; HPSI $=-0.365 / 0)$ : residue $\mathbf{W 1 7 8}$ in the principal subunit interacts with $P 150$ in the complementary subunit. This is the unique interaction left from those constituting the A-A "hot core". In fact, the conserved residues W85, Y148 in the complementary subunit $\mathrm{B}$ are involved in the formation of other interactions with residues of Loop C, i.e. they contribute to keep Loop C close.

3.3.4 The interface B-A. The dimer B-A was extracted from the heteromeric $5-\mathrm{HT}_{3}-\mathrm{R}$ structure. This dimer is characterized by having a $\mathrm{B}$ monomer as the principal subunit and an $\mathrm{A}$ monomer as the complementary subunit. Two B-A interfaces exist 
in the heteropentamer $5-\mathrm{HT}_{3 \mathrm{~A} / \mathrm{B}}-\mathrm{R}$, since a $\mathrm{B}-\mathrm{A}$ interface is formed by the adjacent monomers at positions $\mathbf{c}$ and $\mathbf{a}$ and by the adjacent monomers at positions $\mathbf{d}$ and $\mathbf{e}$ in the pentameric arrangement (Fig. 2c). These interfaces show similar EP and $\mathrm{H}$ distribution, however, the dimer e-d structure has a rmsd from the B-B dimer of $2.07 \AA$, while the corresponding rmsd of the c-a dimer is somewhat higher: $2.32 \AA$. Therefore, the analysis will be performed at the e-d dimer interface.

As highlighted in the previous cases, there are three major areas showing a good electrostatic potential complementarity, although the dimensions of these regions are largely reduced (i.e. they are formed by a lower number of residues) with respect to both the $\mathrm{A}-\mathrm{A}$ and the $\mathrm{A}-\mathrm{B}$ interfaces. These regions are also those showing the larger hydrophobic complementarity, as it can be observed in Fig. 6. Interestingly, no interaction between Loop $\mathrm{C}$ and the complementary subunit can be observed: similarly to the B-B interface, Loop C is in the open conformation, which means the channel is inactive.

Zone $1(\max$ EPSI $=-1.0$; HPSI $=-0.92 /+1)$ : this area is formed by the interaction between the residue D127 in the principal subunit B and K130 in the complementary subunit A. This determines the very high value of the EPSI in this area.

Zone $2(\max$ EPSI $=-1.0$; HPSI $=0.61 /+1)$ : the interacting residues are: E183 in the principal subunit and Q146 in the complementary one.

Zone $3(\max$ EPSI $=-0.98$; HPSI $=-0.7 /+0.9)$ : the interacting residues are E160 and T161 in the principal subunit and Q206 in the complementary subunit.

Of all the residues which have been shown to constitute the "hot centre" in the $5-\mathrm{HT}_{3}-\mathrm{R} \mathrm{A}-\mathrm{A}$ homodimer, ${ }^{26}$ all those which are located at the complementary interface (i.e. Y68, Y83, W85 and Y148) are present also in the B-A heterodimer, however they lack their counterpart on the principal subunit, the main hot spot $\mathbf{W 1 7 8},{ }^{26}$ which is here substituted by the His residue. This causes the loss of an aromatic cluster that forms the "hot core" and is centred at the aromatic residue W178 (in the principal subunit) of the $\mathrm{A}-\mathrm{A}$ interface with a consequent critical destabilization of the interface.

\section{Discussion}

\subsection{The $5-\mathrm{HT}_{3}-\mathrm{R}$ assembling mechanism}

In their recent work, Ortells and Barrantes ${ }^{25}$ have also tackled the problem of the assembly mechanism of the LGICs, by taking into account the various nACh-R subunits. They tried to predict whether the $\beta 1 \delta \alpha 1 \gamma \alpha 1$ receptor assembles in a "heterodimer model" or in the alternative "sequential model". In the first case, the two dimers $\delta \alpha 1$ and $\gamma \alpha 1$ form and then associate with each other and with the remaining $\beta 1$ subunit; in the second case, heterodimers of different compositions are formed to assemble rapidly the $\alpha 1 \beta 1 \gamma$ trimer and subsequently add the $\delta$ and the second $\alpha 1$ subunits.

Determining the correct assembling mechanism of the $5-\mathrm{HT}_{3}-\mathrm{R}$ is not straightforward. However, the analysis of the MEPs of the A and B subunits can help making some preliminary working hypotheses (to be further verified or confuted by experiments) about the reasonable and possible formation of dimers and of the final heteropentamers. On simple electrostatic bases, the dimers AA and BA appear to form with a higher probability than $\mathrm{AB}$ and $\mathrm{BB}$. However, if also the hydrophobic and short range electrostatic interactions at the interface are considered, the $\mathrm{AB}$ interface seems to be more stable than the BA. In addition, the dimer $\mathrm{AB}$ appears to be most reactive dimer since, having a positive complementary interface and a negative principal interface, it can interact with all other subunits or dimers. In contrast, the BA dimer, which has both the interfaces negatively charged, should interact preferably either with the A subunit or with the AB dimer, which both have a positive complementary interface.

Therefore, a few simple electrostatic-based mechanisms to assemble the pentameric receptors can be hypothesized:

(1) Five subunits A assemble one after the other to directly form the homopentameric receptor A or they assemble two by two, to form AA dimers which then assemble together and bind a fifth additional A subunit to get the receptor.

(2) Two AB dimers assemble to form an ABAB tetramer, which successively interact with either an A subunit to form an ABABA pentamer or with a $\mathrm{B}$ subunit to form a BBABA pentamer.

(3) A subunit BA assemble with a subunit $\mathrm{AB}$ to form a tetramer BAAB, which successively binds to a third A subunit to form an ABABA pentamer. In this case, the formation of a BBBAA pentamer seems to be less favoured, at least in simple electrostatic considerations.

\subsection{Comparison of the $5-H^{-} T_{3}-\mathrm{R}$ interfaces}

The analysis of the short range electrostatic and hydrophobic properties of the interfaces which can form the homo- and the heteropentameric 5- $\mathrm{HT}_{3}$ - Rs confirmed the results of a previous study $^{26}$ based on computational alanine scanning, which highlighted the presence at the $\mathrm{A}-\mathrm{A}$ interface of a central aromatic region which stabilizes the interface and therefore named the "hot core". This "hot core" and its local physico-chemical complementarity is completely lost at the B-B interface, where the local sequence and structure are different. Interestingly, the two heterodimeric interfaces, $\mathrm{A}-\mathrm{B}$ and $\mathrm{B}-\mathrm{A}$, show properties which are intermediate between the two homomeric interfaces: at the A-B interface, the "hot core" is partially maintained and the physico-chemical complementarity in the corresponding area is also partially preserved (see Zone 3 in the A-B interface section). The maintained features are due to the presence of $\mathrm{W} 178$ in the A principal subunit. The B-A interface instead is more similar to the $\mathrm{B}-\mathrm{B}$ one: although many residues forming the "hot core" are present on the complementary subunit (which is A subunit), they are not able to form a core aromatic cluster, probably due to the lack of the counterpart residue W178). Thus, in agreement with the results previously found, ${ }^{26}$ the A-B interface seems to be more similar to the $\mathrm{A}-\mathrm{A}$ one and the $\mathrm{B}-\mathrm{A}$ interface seems to be more similar to the B-B one.

This hypothesis is further supported by the observation reported in Section 3.1 regarding the conformation of the homo- and heteropentamers.

Thus, according to the model based on the $2 \mathrm{~A}: 3 \mathrm{~B}$ stoichiometry with a BBABA arrangement, the A-B interface could apparently substitute the $\mathrm{A}-\mathrm{A}$ interface. This conclusion, although being in agreement with the hypothesis by Barrera et al., ${ }^{16}$ disagrees with that of Lummis and co-workers. ${ }^{17,18}$ Actually, these 
Table 1 Amino acid residues at the interface of the homo- and hetero-5-HT $-\mathrm{R}$ interfaces and qualitative predictions on receptor functionality made on the basis of the information extracted from the computational model elaborated

\begin{tabular}{|c|c|c|c|c|c|c|c|c|c|c|c|}
\hline \multirow[b]{2}{*}{ Homo } & \multirow[b]{2}{*}{ Hetero } & \multirow[b]{2}{*}{ Princ. subunit } & \multirow[b]{2}{*}{ Compl. subunit } & \multirow[b]{2}{*}{$\operatorname{pos} 178$} & \multirow[b]{2}{*}{ pos 68} & \multirow[b]{2}{*}{$\operatorname{pos} 83$} & \multirow[b]{2}{*}{ pos 85} & \multirow[b]{2}{*}{ pos 148} & \multirow[b]{2}{*}{$\operatorname{pos} 150$} & \multicolumn{2}{|c|}{ Functional receptor } \\
\hline & & & & & & & & & & Prediction & Exp. evidence \\
\hline $\mathrm{A}-\mathrm{A}$ & & A & A & $W$ & $Y$ & $Y$ & $W$ & $Y$ & $P$ & Yes & 27,46 \\
\hline \multirow[t]{3}{*}{ B-B } & & $\mathrm{B}$ & $\mathrm{B}$ & $\mathrm{H} / \mathrm{I}$ & $\mathrm{H}$ & $\mathrm{S}$ & W & $\mathrm{Y}$ & $\mathrm{P}$ & No & 27,46 \\
\hline & $\mathrm{A}-\mathrm{B}$ & A & B & W & $\mathrm{H}$ & $\mathrm{S}$ & $\mathrm{W}$ & $\mathrm{Y}$ & $\mathrm{P}$ & Maybe YES & \\
\hline & B-A & B & A & $\mathrm{H} / \mathrm{I}$ & $\mathrm{Y}$ & $\mathrm{Y}$ & W & $\mathrm{Y}$ & $\mathrm{P}$ & Probably NOT & \\
\hline \multirow[t]{3}{*}{$\mathrm{C}-\mathrm{C}$} & & $\mathrm{C}$ & $\mathrm{C}$ & $\mathrm{F}$ & $\mathrm{S}$ & $\mathrm{F}$ & W & $\mathrm{D}$ & $\mathrm{P}$ & NO from exp & 46 \\
\hline & $\mathrm{C}-\mathrm{A}$ & $\mathrm{C}$ & A & $\mathrm{F}$ & $\mathrm{Y}$ & $\mathrm{Y}$ & W & $\mathrm{Y}$ & $\mathrm{P}$ & Maybe YES & \\
\hline & $\mathrm{A}-\mathrm{C}$ & A & $\mathrm{C}$ & W & $\mathrm{S}$ & $\mathrm{F}$ & W & D & $\mathrm{P}$ & Probably NOT & \\
\hline \multirow[t]{3}{*}{ D-D } & & $\mathrm{D}$ & $\mathrm{D}$ & $\mathrm{S}$ & $\mathrm{S}$ & - & - & $\mathrm{K}$ & $\mathrm{T}$ & NO from exp & 46 \\
\hline & $\mathrm{D}-\mathrm{A}$ & $\mathrm{D}$ & A & $\mathrm{S}$ & Y & $\mathrm{Y}$ & W & $\mathrm{Y}$ & $\mathrm{P}$ & Probably NOT & \\
\hline & $\mathrm{A}-\mathrm{D}$ & A & $\mathrm{D}$ & $\mathrm{W}$ & $\mathrm{S}$ & - & - & $\mathrm{K}$ & $\mathrm{T}$ & Probably NOT & \\
\hline \multirow[t]{3}{*}{$\mathrm{E}-\mathrm{E}$} & & $\mathrm{E}$ & $\mathrm{E}$ & $\mathrm{F}$ & $\mathrm{S}$ & $\mathrm{F}$ & $\mathrm{W}$ & $\mathrm{K}$ & $\mathrm{P}$ & NO from exp & 46 \\
\hline & $\mathrm{E}-\mathrm{A}$ & $\mathrm{E}$ & A & $\mathrm{F}$ & $\mathrm{Y}$ & $\mathrm{Y}$ & $\mathrm{W}$ & $\mathrm{Y}$ & $\mathrm{P}$ & Maybe YES & \\
\hline & $\mathrm{A}-\mathrm{E}$ & $\mathrm{A}$ & $\mathrm{E}$ & $\mathrm{W}$ & $\mathrm{S}$ & $\mathrm{F}$ & $\mathrm{W}$ & $\mathrm{K}$ & $\mathrm{P}$ & Probably NOT & \\
\hline
\end{tabular}

discrepancies among experiments and modelling studies could coexist in case that different cell types and/or different species express receptors with different stoichiometry or if the same interface in receptors with different compositions and stoichiometries assumes different conformations. A different explanation might be that the receptor behaviour does not depend only on the structure of its extracellular portion, but is instead due to other factors that are not taken into account here, which are connected either to the transmembrane or to the extracellular portion of the system. At present, more experimental information are needed to discriminate between the two principal hypotheses about the $5-\mathrm{HT}_{3}-\mathrm{R}$ stoichiometry and assembly.

Previous works ${ }^{25}$ tried a superficial characterization of the 5- $\mathrm{HT}_{3}-\mathrm{R}$ interfaces $\mathrm{A}-\mathrm{A}, \mathrm{A}-\mathrm{B}, \mathrm{B}-\mathrm{A}$ and $\mathrm{B}-\mathrm{B}$, and concluded that, while all these four dimers can form (at least from a shape complementarity point of view), the $\mathrm{A}-\mathrm{A}$ and $\mathrm{B}-\mathrm{A}$ interfaces should be characterized by stronger interface interactions than $\mathrm{B}-\mathrm{B}$ and $\mathrm{A}-\mathrm{B}$.

These results are in partial disagreement with the results of the present study, which support a model where, although the formation of a B-A interface appears to be favoured from the electrostatic point of view, the A-B interface should behave more similarly to the A-A interface. This discrepancy might be ascribed to the different $5-\mathrm{HT}_{3}-\mathrm{R}$ structural models used: in fact, previous analyses ${ }^{25}$ were based on receptor monomers modelled using the AChBP structure as the template, while here the nAChR structure was used as the template, then pentameric receptor structures were assembled, optimised and used to extract dimeric models. It is also possible that the different conclusions are due to the different computational techniques used: here, the hydrophobic and electrostatic properties of dimeric couples are directly calculated, while previously ${ }^{25}$ property indices were computed which should be able to detect whether single monomer could or could not assemble into a dimer.

To get additional insights into this controversial issue, a much larger number of conformations of each dimer should be sampled and the physico-chemical characterization repeated.

\subsection{Comparison of the $5-\mathrm{HT}_{3}-\mathrm{R}$ subunit sequences $\mathrm{C}, \mathrm{D}$ and $\mathrm{E}$}

In the previous paragraph, the different interfaces found in the functional $5-\mathrm{HT}_{3 \mathrm{~A}}-\mathrm{R}$ and $5-\mathrm{HT}_{3 \mathrm{~A} / \mathrm{B}}-\mathrm{R}$ (with hypothetical stoichiometry BBABA) were analysed to compare their structural and physico-chemical differences within the large structural and sequence composition of this class of receptors. This is nevertheless still a partial picture of the overall potential variability of the $5-\mathrm{HT}_{3}-\mathrm{R}$ composition. In fact, besides the well characterized A and B subunits, at least three more subunits are found, named C, D and E, of which little is known. These other subunits could, at least in principle, form homomeric and heteromeric $5-\mathrm{HT}_{3}-\mathrm{R}$ receptors. However, from experiments, it is known ${ }^{46}$ that, in order to be functional, subunits $\mathrm{C}, \mathrm{D}$, and $\mathrm{E}$ must be co-expressed with subunit A, therefore, no homomeric $\mathrm{C}, \mathrm{D}$, and $\mathrm{E}$ receptor will be considered hereafter.

Of course, further experimental work is required to verify if receptors with $\mathrm{C}, \mathrm{D}$ or $\mathrm{E}$ subunits do exist and if they are active, however, in the meanwhile it would be nevertheless interesting to try to predict whether heterodimeric $5-\mathrm{HT}_{3}-\mathrm{R}$ extracellular interfaces containing monomers $\mathrm{C}, \mathrm{D}$ and $\mathrm{E}$ could be functional or not. To perform this task, models of these $5-\mathrm{HT}_{3}-\mathrm{R}$ sequences would be needed, however, this modelling exercise is not straightforward, since the $5-\mathrm{HT}_{3}-\mathrm{R}$ $\mathrm{C}, \mathrm{D}$ and $\mathrm{E}$ sequences have low sequence identities with the X-ray structures of nACh-R subunits (SeqIds range between 6 and 23, as computed by ClustalW, ${ }^{30,31}$ Fig. 1).

Here, a few hypotheses are worked out on the bases of the only information available about the receptor sequences and taking the moves from the above comparison performed on the interfaces containing subunits $\mathrm{A}$ and $\mathrm{B}$. The interfaces formed by considering only binary heterodimers, i.e. $5-\mathrm{HT}_{3 \mathrm{~A} / \mathrm{D}}-\mathrm{R}$, $5-\mathrm{HT}_{3 \mathrm{~A} / \mathrm{D}}-\mathrm{R}, 5-\mathrm{HT}_{3 \mathrm{~A} / \mathrm{E}}-\mathrm{R}$, are listed in Table 1.

The next assumption is that, in order to be functional, the dimers formed by $5-\mathrm{HT}_{3}-\mathrm{R} \mathrm{C}$ to $\mathrm{E}$ monomers need to have similar physico-chemical properties to those of the A-A dimer. Therefore, the presence of the aromatic hot cluster, and in particular of residue $\mathbf{W 1 7 8}$ in the principal subunit and residues Y83, W85, Y68 and Y148 in the complementary subunit, will be taken as a reference point for functionality.

A further hypothesis is that an interface behaves always in the same way independently of the stoichiometry and composition of the receptor where it is found. For example, since the homopentamer $5-\mathrm{HT}_{3 \mathrm{~B}}-\mathrm{R}$ is not functional, each of its $\mathrm{B}-\mathrm{B}$ interfaces is supposed to be non-functional and therefore, also 
the $\mathrm{B}-\mathrm{B}$ interface found in the $5-\mathrm{HT}_{3 \mathrm{~A} / \mathrm{B}}-\mathrm{R}$ is considered to be non-functional. This assumption may of course be invalidated by significant conformational modification occurring in the heteropentamer, which are not present in the homomeric receptors.

The multiple sequence alignment reported in Fig. 1 allows the comparison of the sequences $\mathrm{A}$ and $\mathrm{B}$ to the sequences $\mathrm{C}$, $\mathrm{D}$ and $\mathrm{E}$ and the translation of the structural and physicochemical information from the first group to second group of sequences (the alignments used to build the structural models of $5-\mathrm{HT}_{3}-\mathrm{R} \mathrm{A}$ and $\mathrm{B}$ shown in a figure reported by Barbosa et $a l .{ }^{27}$ should be considered for comparison).

The first interesting observation is that, while $\mathrm{C}$ and $\mathrm{E}$ are highly similar (SeqI $=74 \%$ ), $\mathrm{D}$ is different from all the other $5-\mathrm{HT}_{3}-\mathrm{R}$ sequences, the most similar sequence being $\mathrm{C}$ with a SeqI of $42 \%$. In fact, D shares only a $15 \%$ and a $21 \%$ sequence similarity with $\mathrm{B}$ and $\mathrm{A}$, respectively: should $\mathrm{D}$ give origin to functional homo- or heteromeric receptors, we can expect these latter to have structural and functional determinants which are different from receptors composed of $\mathrm{A}$ and $\mathrm{B}$ subunits.

W178, which is apparently a key feature of the A-A interface, is not conserved in the $5-\mathrm{HT}_{3}-\mathrm{R} \mathrm{D}$ sequence, where at this position a Ser is located, which cannot maintain the aromatic interaction network formed by Trp (see Table 1). At this same position (178), Phe is found in both $5-\mathrm{HT}_{3}-\mathrm{R}$ subunits $\mathrm{C}$ and $\mathrm{E}$ : Phe may partially retain the properties of Trp and its capability of forming $\pi-\pi$ stacking networks, while it has no capabilities of establishing polar interactions. W178 is not even present in the $5-\mathrm{HT}_{3}-\mathrm{R}$ subunit $\mathrm{B}$ model, where it is structurally substituted by a His, which seems not to be able to maintain the interaction network of Trp, as showed by a recent Molecular Dynamics simulations study by De Rienzo et al. ${ }^{26}$ In the multiple alignment of the $5-\mathrm{HT}_{3}-\mathrm{R}$ subunit sequences, $\mathbf{W 1 7 8}$ of subunit $\mathrm{A}$ is substituted by Ile in subunit $\mathrm{B}$ : this residue is even less suited than His to keep the local aromatic network.

On the complementary surface, the hot spot Y83 (in the A subunit) ${ }^{26}$ is substituted by Ser in the B subunit, which preserves only the polar features of Trp, while both in $\mathrm{C}$ and $\mathrm{E}$ it is replaced by Phe, which potentially retains only the aromatic features. Interestingly, in sequence D, position $\mathbf{1 7 8}$ is represented by a gap, which does not allow us to make any hypothesis about the protein structure in that region. While in all the other $5-\mathrm{HT}_{3}-\mathrm{R}$ subunit sequences $\boldsymbol{P 1 5 0}$ is conserved, in subunit D it is substituted by a Thr: since $\boldsymbol{P 1 5 0}$ in subunit A is known to be determinant for the receptor functionality, its absence is a further hint of the incapability of this subunit D sequence to be functional.

The complementary subunit warm spot ${ }^{26} \mathrm{~W} 85$ is conserved in all the sequences with the unique exception of $5-\mathrm{HT}_{3}-\mathrm{R} \mathrm{D}$, where the sequence alignment shows the presence of a His, which is nevertheless polar and aromatic, although smaller than Trp.

Position 68, which is occupied by Tyr in principal subunit A, is occupied by Ser in all the other sequences exception made for $5-\mathrm{HT}_{3}-\mathrm{R} \mathrm{B}$, where it is His. The common feature is the polarity, however only His retains the aromatic features of Tyr.

Finally, residue Y148 in the principal surface A is conserved only in subunit B, while it is replaced by Asp in sequence $\mathrm{C}$ and by Lys in both the D and E sequences. Both Asp and Lys can form H-bonds with nearby residues, but they destabilize the aromatic hot cluster. Substitution of Y148 at the A-A interface with Lys would enlarge the positive electrostatic potential area on Zone 1 in the complementary surface area, thus increasing the electrostatic complementarity, while the Asp would modify the electrostatic potential making it more negative and diminishing the local electrostatic complementary. In both cases (with Lys and Asp), the hydrophobic complementarity would be diminished. Mutations of this kind would reasonably modify the interface functionality, making it less active.

Thus, as it is summarized in Table 1, from this analysis based on the physico-chemical properties of the interfaces and on previous CASM studies, ${ }^{26}$ only a few of the dimers studied should be functional. In particular, considering as fundamental the presence of Trp at position $\mathbf{1 7 8}$ at the principal interface and searching for the largest similarities between the various interfaces and the $\mathrm{A}-\mathrm{A}$ interface, only the $\mathrm{C}-\mathrm{A}$ and $\mathrm{E}-\mathrm{A}$ interfaces appear to be able to build functional receptors: here, the complementary interfaces are the same as in the A-A dimer and W178 is replaced by a Phe, which can still stabilize the hot core $\pi-\pi$ network. Instead the $\mathrm{A}-\mathrm{E}$ and $\mathrm{A}-\mathrm{C}$ interfaces which have a complementary surface identical to the A-A dimer will reasonably be nonfunctional (or at least less functional) due to the presence of a charged residue (Asp or Lys) which lowers the local surfacesurface physico-chemical complementarity. Similarly, also the homodimer $\mathrm{E}-\mathrm{E}$ and $\mathrm{C}-\mathrm{C}$ can be considered as non-functional interfaces. Finally, none of the dimers built by the $5-\mathrm{HT}_{3}-\mathrm{R} \mathrm{D}$ sequence appears to be functional, due to very different sequence of this monomer in the local interface area.

\section{Conclusions}

Here, the long-range interaction features of subunits $5-\mathrm{HT}_{3}-\mathrm{R}$ $\mathrm{A}$ and $\mathrm{B}$ and of their possible combinations into dimers are analysed together with the physico-chemical properties of the various putative $5-\mathrm{HT}_{3}-\mathrm{R}$ interfaces, on the bases of the structural conformations of the models and of the known sequences, in order to develop working hypotheses about the stoichiometries and functionalities of the receptors.

The results confirmed the presence of an aromatic cluster, located in the core of the $\mathrm{A}-\mathrm{A}$ interface, as the key determinant of the monomer-monomer stability, in agreement with previous studies. This cluster is completely absent in the B-B dimer which is known to give origin to non-functional homopentameric 5-HT $\mathrm{H}_{3}$-Rs. On this basis, among all the other possible interfaces constituted by the known $5-\mathrm{HT}_{3}-\mathrm{R}$ sequences $\mathrm{A}, \mathrm{B}, \mathrm{C}, \mathrm{D}$, and $\mathrm{E}$ only the $\mathrm{C}-\mathrm{A}$ and $\mathrm{E}-\mathrm{A}$ interfaces are predicted to be able to build functional receptors. Further experimental analyses will be necessary to shed new light on this complex issue.

\section{References}

1 J. Hannon and D. Hoyer, Behav. Brain Res., 2008, 195, 198-213.

2 J. A. Peters, T. G. Hales and J. J. Lambert, Trends Pharmacol. Sci., 2005, 26, 587-594.

3 N. M. Barnes, T. G. Hales, S. C. R. Lummis and J. A. Peters, Neuropharmacology, 2009, 56, 273-284 (and references therein).

4 F. G. Boess, S. C. R. Lummis and I. L. Martin, J. Neurochem., 1992, 59, 1692-1701. 
5 F. G. Boess, R. Beroukhim and I. L. Martin, J. Neurochem., 1995, 64, 1401-1405.

6 K. L. Price, K. S. Bower, A. J. Thompson, H. A. Lester, D. A. Dougherty and S. C. R. Lummis, Biochemistry, 2008, 47, 6370-6377.

7 J. A. Peters, T. G. Hales and J. J. Lambert, Trends Pharmacol. Sci., 2005, 26, 587-594.

8 A. Karlin, Nat. Rev. Neurosci., 2002, 3, 102-114.

9 D. C. Reeves and S. C. R. Lummis, Mol. Membr. Biol., 2002, 9, $11-26$.

10 H. A. Lester, M. I. Dibas, D. S. Dahan, J. F. Leite and D. A. Dougherty, Trends Neurosci., 2004, 27, 329-336.

11 N. Unwin, J. Mol. Biol., 2005, 346, 967-989.

12 S. M. Sine and A. G. Engel, Nature, 2006, 440, 448-455.

13 A. J. Thompson and S. C. R. Lummis, Curr. Pharm. Des., 2006, 12, 3615-3630 (and references therein).

14 A. J. Thompson and S. C. R. Lummis, Expert Opin. Ther. Targets, 2007, 11, 527-540 (and references therein).

15 B. Niesler, B. Frank, J. Kapeller and G. A. Rappold, Gene, 2003, 310, 101-111.

16 N. P. Barrera, P. Herbert, R. M. Henderson, I. L. Martin and J. M. Edwardson, Proc. Natl. Acad. Sci. U. S. A., 2005, 102, 12595-12600 (and references therein).

17 M. Lochner and S. R. C. Lummis, Biophys. J., 2010, 98, 1494-1502.

18 A. J. Thompson, K. L. Price and S. C. R. Lummis, J. Physiol., 2011, 589, 4243-4257.

19 N. Yaakob, D. T. Malone, B. Exintaris and H. R. Irving, Curr. Mol. Med., 2011, 11, 57-68.

20 A. J. Thompson, H. A. Lester and S. C. R. Lummis, Q. Rev. Biophys., 2010, 43, 449-499.

21 B. Niesler, J. Walstab, S. Combrink, D. Moeller, J. Kapeller, J. Rietdorf, M. Göthert, H. Bönisch, G. Rappold and M. Brüss, Mol. Pharmacol., 2007, 72(1), 8-17.

22 K. S. Bower, K. L. Price, L. E. Sturdee, M. Dayrell, D. A. Dougherty and S. C. R. Lummis, Eur. J. Pharmacol., 2008, 580, 291-297.

23 N. S. Millar, Biochem. Soc. Trans., 2003, 31, 869-874.

24 X. M. Xu and Z. W. Hall, Nature, 1991, 352, 64-67.

25 M. O. Ortells and G. E. Barrantes, Proteins, 2008, 70, 473-488.

26 F. De Rienzo, A. J. Moura Barbosa, M. A. S. Perez, P. A. Fernandes, M. J. Ramos and M. C. Menziani, J. Biomol. Struct. Dyn., 2012, 30, 267-284.

27 A. J. Moura Barbosa, F. De Rienzo, M. J. Ramos and M. C. Menziani, Eur. J. Med. Chem., 2010, 45, 4746-4760.
28 The UniProt Consortium, The Universal Protein Resource (UniProt), Nucleic Acids Res, 2008, 36, D190-D195.

29 P. R. Joshi, A. Suryanarayanan, E. Hazai, M. K. Schulte, G. Maksay and Z. Bikádi, Biochemistry, 2006, 45, 1099-1105.

30 M. A. Larkin, G. Blackshields, N. P. Brown, R. Chenna, P. A. McGettigan, H. McWilliam, F. Valentin, I. M. Wallace, A. Wilm, R. Lopez, J. D. Thompson, T. J. Gibson and D. G. Higgins, Bioinformatics, 2007, 23, 2947-2948.

31 M. Goujon, H. McWilliam, W. Li, F. Valentin, S. Squizzato, J. Paern and R. Lopez, Nucleic Acids Res., 2010, 38(Suppl), W695-W699.

32 A. Sali and T. L. Blundell, J. Mol. Biol., 1993, 234, 779-815.

33 R. A. Laskowski, M. W. MacArthur, D. S. Moss and J. M. Thornton, J. Appl. Crystallogr., 1993, 26, 283-291.

34 QUANTA, Molecular Simulations, San Diego, CA, U. S. A.

35 G. Vriend, J. Mol. Graphics, 1990, 8, 52-56.

36 R. W. W. Hooft, G. Vriend, C. Sander and E. E. Abola, Nature, 1996, 381, 272.

37 B. R. Brooks, R. E. Bruccoleri, B. D. Olafson, D. J. States, S. Swaminathan and M. Karplus, J. Comput. Chem., 1983, 4, 187-217.

38 A. D. J. MacKerell, D. Bashford, M. Bellott, R. L. J. Dunbrack, J. D. Evanseck, K. J. Field, S. Fischer, J. Gao, H. Guo, S. Ha, D. Joseph-McCarthy, L. Kuchnir, K. Kuczera, F. T. K. Lau, C. Mattos, S. Michnick, T. Ngo, D. T. Nguyen, B. Prodhom, W. E. Reiher III, B. Roux, M. Schlenkrich, J. C. Smith, R. Stote, J. Straub, M. Watanabe, J. Wiorkiewicz-Kuczera, D. Yin and M. Karplus, J. Phys. Chem. B, 1998, 102, 3586-3616.

39 R. R. Gabdoulline, R. C. Wade and D. Walther, Nucleic Acids Res., 2003, 31, 3349-3351.

40 R. R. Gabdoulline, R. C. Wade and D. Walther, Trends Biochem. Sci., 1999, 24, 285-287.

41 MolSurfer 1.2. http://projects.villa-bosch.de/dbase/molsurfer/.

42 J. D. Madura, J. M. Briggs, R. C. Wade, R. Davis, B. A. Luty, A. Ilin, J. Antonsiewicz, M. K. Bagheri, B. Scott and J. A. McCammon, Comput. Phys. Commun., 1995, 91, 57-95.

43 T. J. Dolinsky, J. E. Nielsen, J. A. McCammon and N. A. Baker, Nucleic Acids Res., 2004, 32, W665-W667.

44 PDB2PQR Server. http://kryptonite.nbcr.net/pdb2pqr/.

45 D. Eisenberg, R. M. Weiss, T. C. Terwilliger and W. Wilcox, Faraday Symp. Chem. Soc., 1982, 17, 109-120.

46 A. A. Jensen, P. A. Davies, H. Brauner-Osborne and K. Krzywkowski, Trends Pharmacol. Sci., 2008, 29, 437-444. 